\title{
Distinct TPR motifs of Cyc8 are involved in recruiting the Cyc8-Tup1 corepressor complex to differentially regulated promoters
}

\author{
Dimitris Tzamarias ${ }^{1}$ and Kevin Struhl ${ }^{2}$ \\ Department of Biological Chemistry and Molecular Pharmacology, Harvard Medical School, Boston, Massachusetts 02115
}

The yeast Cyc8(Ssn6)-Tup1 complex is required for transcriptional repression of distinct sets of genes that are regulated by glucose, oxygen, cell type, and DNA damage. It has been proposed that the Cyc8-Tup1 complex is a corepressor that is recruited to promoters by interacting with pathway-specific DNA-binding proteins. Previously, we showed that a specific region of Tup1 mediates the general transcriptional repression function of the complex. Here, we define functional domains of $\mathrm{Cyc8}$, a protein consisting primarily of 10 tandem copies of a TPR motif. Distinct combinations of TPR motifs are required specifically for direct interaction with Tup1, repression of oxygen-regulated genes, and repression of glucose-regulated genes. In contrast, the WD motifs of Tup1 are not essential for repression of genes regulated by glucose and oxygen, but they are required for those regulated by cell type and DNA damage. In addition, we show that the Cyc8-Tup1 complex functions both as a corepressor and an inhibitor of Mig1, a protein that binds to promoters of glucose-repressible genes. These observations suggest that different Cyc8 TPR motifs and the Tup1 WD domain mediate distinct protein-protein interactions that link the Cyc8-Tup1 corepressor to structurally dissimilar DNA-binding proteins required for pathway-specific regulation.

[Key Words: TPR motifs; Cyc8(Ssn6)-Tupl complex; transcriptional repression; corepressor; eukaryotic gene regulation]

Received January 16, 1995; revised version accepted February 28, 1995.

Eukaryotic organisms have a variety of global repression mechanisms that negatively regulate the transcription of many apparently unrelated genes. One such mechanism, chromatin-based repression, involves histones (Grunstein, 1990) and nonhistone proteins such as Spt4-6 (Swanson and Winston 1992). A second global repression mechanism involves proteins that interfere directly with components of the basic transcription machinery. For example, human Drl (Inostroza et al. 1992) and yeast Motl (Auble et al. 1994) block the interaction of the TATA-binding protein with the TATA element, and the yeast NOT complex differentially affects TATA element utilization (Collart and Struhl 1994). Although chromatin and direct inhibitors of basic transcription factors should repress all genes, there is considerable variation in the extent to which individual genes are affected. In both cases, however, there is no clear pattern that distinguishes genes that are strongly repressed from those that are not affected. In contrast, there is a third mechanism, exemplified by the yeast Cyc8-Tup1 (see below)

\footnotetext{
${ }^{1}$ Present address: Institute of Molecular Biology and Biotechnology, FORTH-HELLAS, Heraklion 711 10, Crete, Greece.

${ }^{2}$ Corresponding author.
}

and the Drosophila polycomb (Paro 1990) complexes, in which specific but apparently unrelated classes of genes are repressed. This more specific form of global repression has the potential to coordinate the regulation of distinct biological pathways.

Cyc8(Ssn6) and Tup1 are physically associated proteins (Williams et al. 1991) that are required for repression of at least four unrelated classes of yeast genes. These include genes regulated by cell type (a-specific and haploid-specific) (Mukai et al. 1991; Keleher et al. 1992), glucose (Schultz and Carbon 1987; Trumbly 1992), oxygen (Zitomer and Lowry 1992), and DNA damage (Elledge et al. 1993). Although the Cyc8-Tup1 complex does not directly bind to promoter sequences, repression of these distinct classes of genes does require pathway-specific DNA-binding proteins. For example, Migl (Nehlin and Ronne 1990), Rox1 (Balasubramanian et al. 1993), and $\alpha 2$ in combination with Mcm 1 (Keleher et al. 1988) or al (Goutte and Johnson 1988) bind, respectively, to the promoters of glucose, oxygen, and cell typeregulated genes. The Cyc8-Tupl complex is clearly involved in $\alpha 2$-dependent repression (Keleher et al. 1992), whereas the evidence for its role in Migl- and Roxldependent repression is only circumstantial. 
Several observations strongly suggest that the Cyc8Tup1 complex is a corepressor that is recruited to promoters via pathway-specific DNA-binding proteins, whereupon it then represses transcription (Keleher et al. 1992). First, $\alpha 2 / \mathrm{Mcml}$, the best characterized DNAbinding repressor, binds to its operator in vivo even in the absence of Cyc8-Tupl, but this binding is not sufficient for repression (Keleher et al. 1992). Second, artificial promoters bearing a 1- $\alpha 2$ or $\alpha 2-\mathrm{Mcm} 1$ operators upstream of heterologous upstream activating sequences (UASs) are strongly repressed in a Cyc8-Tupl-dependent manner, indicating that the Cyc8-Tup1 complex does not inhibit the function of specific activators (Keleher et al. 1992). Third, $\alpha 2$ directly interacts with Tup1, and this interaction is required for repression of cell type-specific genes (Komachi et al. 1994). Fourth, although Cyc8 and Tup1 do not bind directly to DNA, both proteins strongly repress transcription when bound upstream of a functional promoter via the LexA DNA-binding domain (Keleher et al. 1992; Tzamarias and struhl 1994). Such negative regulation at a distance is characteristic of glucose and cell type repression (Johnson and Herskowitz 1985; Struhl 1985), and it suggests that the Cyc8-Tup1 complex inhibits transcription by an active mechanism rather than by steric hindrance.

Analysis of LexA hybrid proteins indicates that the transcriptional repression function of the Cyc8-Tup1 complex is mediated by a specific domain of Tupl (Tzamarias and Struhl 1994). When tethered upstream of a promoter, this Tup1 repression domain does not require Cyc8 to inhibit transcription. Moreover, this domain is required for repression of genes regulated by glucose (SUC2) and oxygen (ANB1). Within this domain, short nonoverlapping regions with minimal sequence similarity can mediate the repression function independently. Although the nature of the repression mechanism is unknown, there is evidence both for effects on the chromatin template (Cooper et al. 1994) and on the basic transcription machinery (Herschbach et al. 1994; Tzamarias and Struhl 1994).

In addition to this repression domain, Tupl contains a separable amino-terminal region (residues 1-72) that directly interacts with Cyc8 (Tzamarias and Struhl 1994) and a carboxy-terminal domain that contains six copies of a WD (also known as $\beta$-transducin) sequence motif (Williams and Trumbly 1990). WD repeats are present in many proteins that are involved in diverse cellular processes, and they have been suggested to mediate proteinprotein interactions. The role of the Tup $\mathrm{WD}$ domain is complex. The Tupl WD motifs interact directly with $\alpha 2$ and are required for repression of cell type genes (Komachi et al. 1994), and some mutations in the WD domain appear to abolish Tupl function (Williams and Trumbly 1990; Tzamarias and Struhl 1994). However, a derivative that completely lacks the WD domain can repress SUC2 and $A N B 1$ transcription and mediate other Tup1 activities (Tzamarias and Struhl 1994).

Although repression of cell type genes involves the direct interaction of $\alpha 2$ with the Tup1 WD motifs (Komachi et al. 1994), the mechanisms by which the Cyc8-
Tupl corepressor is recruited to other classes of promoters is unknown. In this regard, there are no obvious structural similarities among $\alpha 2$, Migl, and Rox1. Previously, we suggested that Cyc8 might be involved in the recruitment process based on the facts that Cyc8 and the Cyc8-interaction domain are not required for repression by LexA-Tup1, but both are essential for repression of SUC2 and ANB1 (Tzamarias and Struhl 1994). The functionally important region of $\mathrm{Cyc} 8$ contains 10 copies of a 34-amino-acid TPR motif (specific motifs are defined herein as TPR1-10 starting from the amino-terminus) (Schultz et al. 1990). As is the case for WD motifs, TPR motifs are present in functionally diverse proteins that are often associated in protein complexes, and they have been proposed to mediate protein-protein interactions (Hirano et al. 1990; Sikorski et al. 1990; Goebl and Yanagida 1991; Lamb et al. 1994). However, there is no evidence that TPR motifs interact directly with other proteins, and their specific biochemical or physiological functions are unknown.

Here, we perform a functional analysis of Cyc8 by assaying a set of deletion mutations for interaction with Tupl and for repression of natural and artificial promoters. We demonstrate that distinct combinations of TPR motifs are required specifically for direct interaction with Tupl, repression of oxygen-regulated genes, and repression of glucose-regulated genes. In addition, we show that Migl is a functional target of the Cyc8-Tupl complex and that Cyc8-Tupl functions both as a corepressor and as a transcriptional inhibitor of Migl. We propose that different Cyc8 TPR motifs and the Tup1 WD domain mediate distinct protein-protein interactions whose cooperative function recruits the corepressor complex to pathway-specific promoters that are recognized by structurally dissimilar DNA-binding proteins.

\section{Results}

\section{A specific region of the Cyc8 TPR domain} associates directly with Tup1

The two-hybrid assay for protein-protein interactions was employed to map the region of Cyc8 that interacts with Tupl (Table 1; Fig. 1). LexA-Cyc8 derivatives were introduced into a yeast strain that expresses a hybrid protein containing Tupl residues 1-72 fused to the VP16 transcriptional activation domain. This region of Tup 1 is necessary and sufficient for interacting with $\mathrm{Cyc} 8$, but it does not mediate transcriptional repression (Tzamarias and Struhl 1994). Interaction between Tupl and Cyc8 regions in the hybrid proteins generates a transcriptional activator that stimulates a target promoter containing four LexA operators upstream of the GAL1 TATA element.

As expected, the combination of Tup1N72-VP16 and LexA-Cyc8 confers 11-fold higher expression levels than either protein alone. Similar or even higher activation was observed with all derivatives that include TPR motifs 1-3 (N175, N300, N351, N597, and N816). In contrast, derivatives containing TPR1 (e.g., N98) or TPR2-7 
Table 1. Two-hybrid assays for Cyc8-Tup1 interaction

\begin{tabular}{|c|c|c|c|c|c|c|c|c|}
\hline \multirow{3}{*}{$\begin{array}{l}\text { LexA hybrid } \\
\text { proteins }\end{array}$} & \multirow{2}{*}{\multicolumn{2}{|c|}{ Fold activation }} & \multicolumn{6}{|c|}{ VP16 hybrid proteins (chromosomal CYC8 allele) } \\
\hline & & & \multirow{2}{*}{$\begin{array}{l}\text { VP16 } \\
\text { (WT) }\end{array}$} & \multirow{2}{*}{$\begin{array}{l}\text { Tup1 } \\
\text { (WT) }\end{array}$} & \multirow{2}{*}{$\begin{array}{l}\text { Tup-VP16 } \\
\text { (WT) }\end{array}$} & \multirow{2}{*}{$\begin{array}{l}\text { VP16 } \\
|c y c 8|\end{array}$} & \multirow{2}{*}{$\begin{array}{l}\text { Tup1 } \\
\text { (cyc8) }\end{array}$} & \multirow{2}{*}{$\begin{array}{c}\text { Tup-VP16 } \\
\text { (cyc8) }\end{array}$} \\
\hline & $(\mathrm{WT})$ & $(\operatorname{cyc} 8)$ & & & & & & \\
\hline Cyc8 & 11 & 8.8 & 4 & 4 & 43 & 2 & 4 & 35 \\
\hline Cyc8-N816 & 24 & 15 & 6 & 4 & 95 & 3 & 4 & 61 \\
\hline Cyc8-N597 & 45 & 63 & 5 & 10 & 450 & 3 & 4 & 250 \\
\hline Cyc8-N351 & 36 & & 7 & 10 & 360 & & & \\
\hline Cyc8-N300 & 40 & & 7 & 6 & 240 & & & \\
\hline Cyc8-N175 & 22 & 32 & 4 & 6 & 130 & 4 & 3 & 95 \\
\hline Cyc8-N98 & 2.4 & 1.7 & 6 & 7 & 17 & 2 & 4 & 7 \\
\hline Сyc8-(79-300) & 1.7 & 2.5 & 4 & 6 & 10 & 3 & 4 & 10 \\
\hline Cyc8-(113-300) & 1.2 & & 4 & 5 & 6 & & & \\
\hline LexA & 1 & 1.7 & 6 & 5 & 5 & 3 & 3 & 5 \\
\hline Vector & 1 & 2.5 & 6 & 5 & 5 & 3 & 2 & 5 \\
\hline
\end{tabular}

$\beta$-Galactosidase activities (average of three independent transformants) of wild-type (WT) or cyc8 deletion strains expressing the indicated proteins. The $l a c Z$ reporter plasmid contains four LexA operators upstream of the GAL1 TATA element. Values are normalized to $A_{600}$ of cells at the time of collecting and are accurate to $\pm 30 \%$. Fold activation represents the ratio of $\beta$-galactosidase activities in strains containing Tupl-VP16 vs. those containing Tup1. Nonrecorded entries indicate that the experiment was not performed.

(79-300) do not confer higher expression levels than that conferred by the LexA control. In all cases, transcriptional stimulation was specific for Tup1N72-VP16; it was not observed with Tup1N72 or the VP16 activation domain alone. Similar results were obtained in $c y c 8$ or tup1 deletion strains, indicating that the observed interactions are not affected significantly by the the presence of wild-type Cyc8 or Tup1. These results demonstrate that the amino-terminal 175 residues of Cyc8 (TPR1-3) are necessary and sufficient for formation of the Cyc8Tupl complex in vivo.

To determine whether this two-hybrid interaction reflected direct contact between the proteins, we examined whether Cyc8-N175 could associate with Tup1 in vitro in the absence of additional yeast proteins (Fig. 2).
Agarose beads containing glutathione $S$-transferase (GST) or GST-Tup 1 were tested for interaction with in vitro-labeled Cyc8-N175 or Cyc8-(79-300). In accord with the results from the two-hybrid assay, GST-Tupl, but not GST, strongly interacts with Cyc8-N175, whereas no interaction is observed between GST-Tup1 and Cyc8-(79-300). Similar results were obtained using GST-Tupl-N72 (data not shown). These results indicate that there is a direct protein-protein interaction between the amino terminal 72 residues of Tupl and the amino terminal residues of Cyc8 (TPR1-3).

\section{Cyc8 represses transcription by recruiting Tup1}

The various LexA-Cyc 8 derivatives were analyzed for

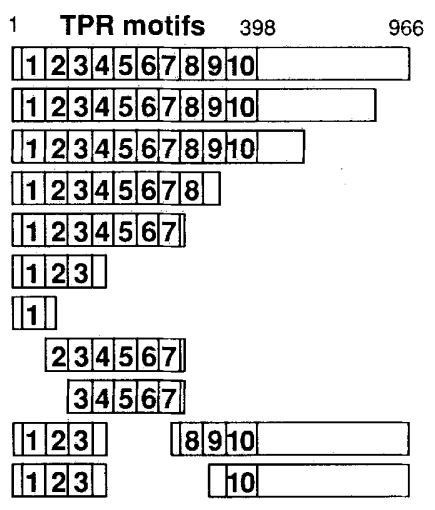

Cyc8
N816
N597
N351
N300
N175
N98
$79-300$
$113-300$
$\Delta 175-281$
$\Delta 175-304$

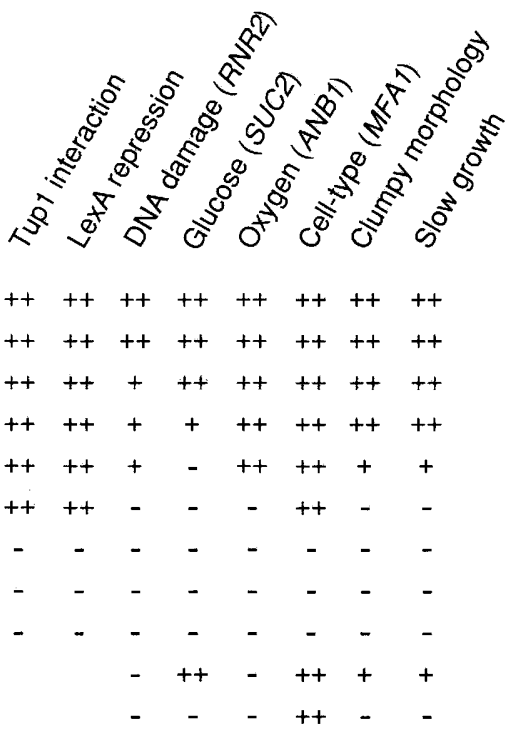

Figure 1. Structure and function of Cyc8 deletion derivatives. The structures of Cyc8 (966 amino acids, including 10 copies of a TPR motif numbered from the amino terminus) and deletion derivatives are indicated along with the intact TPR units that are present. For each derivative, the following phenotypic properties are indicated: Tupl-interaction (Table 1); LexA-dependent repression (Table 2); repression of genes regulated by DNA damage (RNR2), glucose (SUC2), oxygen (ANB1), and cell type (MFA1); and slow growth and clumpy colony morphology (determined by inspection). Phenotypes are defined as follows: $1++1$ Functionally indistinguishable from wild-type allele (CYC8); (+) partial function; $(-)$ functionally indistinguishable from $c y c 8$ deletion allele. 


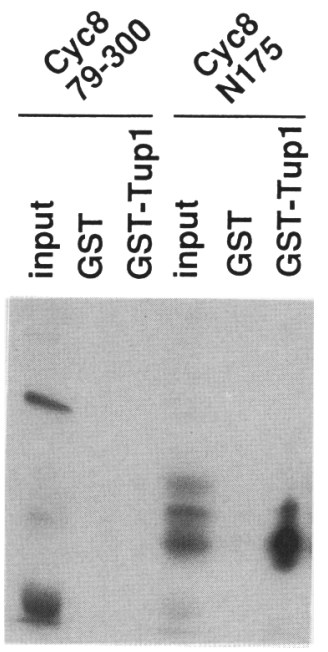

Figure 2. Cyc8-Tupl interaction in vitro. ${ }^{35}$ S-Labeled Cyc8(79-300) or Cyc8-N175 stably bound to agarose beads containing either GST-Tupl or GST alone. Lanes labeled input contain only $20 \%$ of the amount of protein that was incubated with the beads.

transcriptional repression of an artificial promoter that bears four LexA-binding sites upstream of the CYC1 UAS and TATA elements (Table 2; Fig. 1). As reported previously (Keleher et al. 1992; Tzamarias and Struhl 1994), LexA-Cyc8 represses transcription 27 -fold in a Tupl-dependent manner. All LexA-Cyc8 derivatives interacting with Tupl (i.e., that contain $\geqslant 175$ amino-terminal residues) also repress transcription, although some of them are slightly less efficient than LexA-Cyc8. However, LexA-Cyc8 derivatives that fail to interact with Tup1 (e.g., Cyc8 residues 1-98 or 79-300) do not confer repression. Similar results are obtained by testing these constructs in a $c y c 8$ deletion strain. In a tup1 deletion strain, repression by the LexA-Cyc8 derivatives does not occur, and unexpectedly, several of the deleted derivatives actually activate transcription. Thus, when tethered upstream of a functional promoter, the Tupl-interaction domain of Cyc8 (TPR1-3) is necessary and sufficient for Tup1-dependent repression. This observation suggests that LexA-Cyc8 represses transcription simply by recruiting Tupl.

\section{Distinct TPR motifs of Cyc 8 are required for repression of pathway-specific genes}

After removing the LexA domain, we tested the ability of the above Cyc 8 deletion derivatives to repress transcription of genes representing the four regulatory pathways affected by Cyc8-Tup1: cell type (MFA1); glucose (SUC2); oxygen (ANB1); and DNA damage (RNR2). All of these genes are highly expressed in a cyc8 or tup1 deletion strain, but they are silent (or expressed at a low level in the case of RNR2) in a wild-type strain (Fig. 3). The 150 carboxy-terminal residues of Cyc 8 do not appear to be functionally important because the N816 derivative behaves indistinguishably from the wild-type protein. In contrast, derivatives that fail to interact with Tupl (N98, 79-300, 113-300) are unable to repress transcription of any of these genes.

The most interesting class of Cyc 8 derivatives are those carboxy-terminal deletions that differentially affect the pathway-specific genes. Cyc8-N597, a derivative containing the entire TPR domain but lacking the carboxy-terminal 369 residues, efficiently represses $M F A 1, A N B 1$, and SUC2 but only partially represses RNR2. Cyc8-N351, which contains TPR 1-8 and most of TPR9, completely represses MFA1 and ANB1 but only partially represses SUC2 and RNR2. Cyc8-N300, which contains TPR1-7, represses MFA1 and ANB1 but not RNR2 and SUC2. Finally, Cyc8-N175, a derivative that

Table 2. Transcriptional repression by LexA-Cyc8 derivatives

\begin{tabular}{|c|c|c|c|c|c|c|c|c|c|}
\hline \multirow{3}{*}{$\begin{array}{l}\text { LexA hybrid } \\
\text { proteins }\end{array}$} & \multirow{2}{*}{\multicolumn{3}{|c|}{ Fold repression }} & \multicolumn{6}{|c|}{ Promoter (strain background) } \\
\hline & & & & \multirow{2}{*}{$\begin{array}{c}\text { - Lop } \\
\text { (WT) }\end{array}$} & \multirow{2}{*}{$\begin{array}{l}\text { 4 Lop } \\
\text { (WT) }\end{array}$} & \multirow{2}{*}{$\begin{array}{l}- \text { Lop } \\
(c y c 8)\end{array}$} & \multirow{2}{*}{$\begin{array}{l}4 \text { Lop } \\
\text { (cyc8) }\end{array}$} & \multirow{2}{*}{$\begin{array}{l}\text { - Lop } \\
\text { (tup1) }\end{array}$} & \multirow{2}{*}{$\begin{array}{l}4 \text { Lop } \\
\mid \text { tup } 1\end{array}$} \\
\hline & $(\mathrm{WT})$ & $(\operatorname{cyc} 8)$ & (tup1) & & & & & & \\
\hline Cyc8 & 27 & 29 & 2.4 & 60 & 2.3 & 58 & 2.0 & 29 & 12 \\
\hline Cyc8-N816 & 25 & 30 & 0.6 & 85 & 3.4 & 62 & 2.1 & 42 & 64 \\
\hline Cyc8-N597 & 21 & 16 & 0.7 & 92 & 4.3 & 55 & 3.5 & 47 & 65 \\
\hline Cyc8-N351 & 32 & 26 & 0.4 & 100 & 3.1 & 57 & 2.2 & 42 & 97 \\
\hline Cyc8-N300 & 30 & 13 & 0.4 & 85 & 2.8 & 51 & 4.0 & 39 & 85 \\
\hline Cyc8-N175 & 12 & 15 & 1.0 & 80 & 6.6 & 46 & 3.1 & 57 & 58 \\
\hline Cyc8-N98 & 1.8 & 1.9 & 0.8 & 84 & 46 & 48 & 25 & 61 & 74 \\
\hline Cyc8- $-79-300)$ & 0.9 & 0.9 & 0.9 & 64 & 70 & 49 & 52 & 60 & 68 \\
\hline Cyc8-(113-300) & 1.0 & 1.0 & 0.8 & 72 & 71 & 55 & 54 & 55 & 69 \\
\hline $\operatorname{Lex} A$ & 1.2 & 1.1 & 1.3 & 100 & 80 & 55 & 50 & 90 & 70 \\
\hline Vector & 1.2 & 1.0 & 1.3 & 100 & 85 & 55 & 55 & 90 & 70 \\
\hline
\end{tabular}

$\beta$-Galactosidase activities (average of three independent transformants) of wild-type (WT), cyc8, or tup1 strains containing the indicated promoters and expressing the indicated proteins. Values are normalized to $A_{600}$ of cells at the time of collecting and are accurate to $\pm 30 \%$. Fold repression represents the ratio of $\beta$-galactosidase activities in strains containing plasmids that either lack (- Lop) or contain four LexA operators (4 Lop) upstream of the CYC1 promoter fused to the lacZ structural gene. 


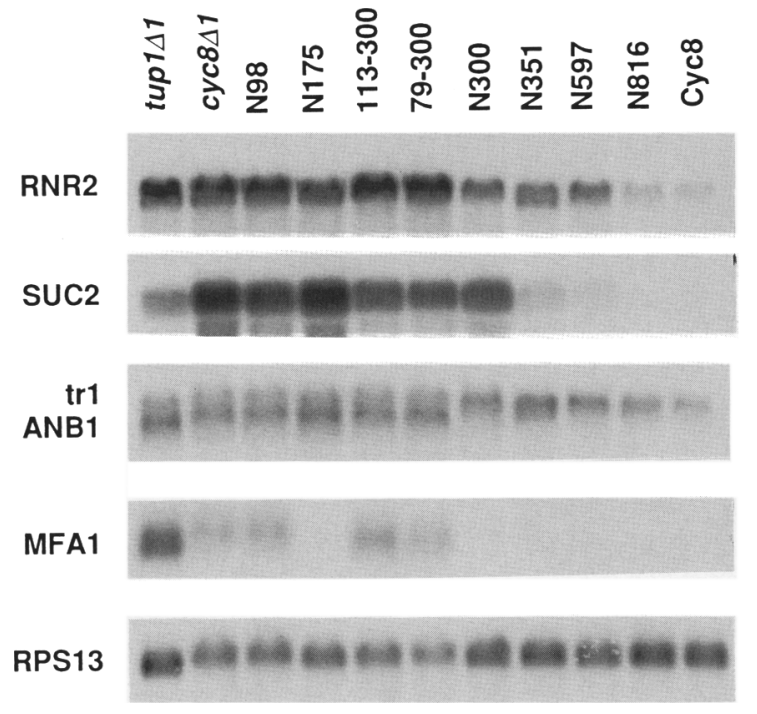

tr1

ANB1

MFA1

RPS13

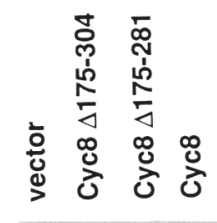

SUC2
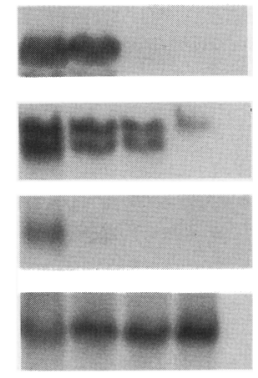

Figure 3. Transcriptional repression of RNR2, SUC2, ANB1, and MFA1 genes by various $C y c 8$ derivatives. RNA from $c y c 8$ deletion strains transformed by the indicated Cyc8 derivatives was fractionated in $1.4 \%$ agarose-formaldehyde gel, transferred to a nylon membrane, and hybridized with ${ }^{32} \mathrm{P}$-labeled probes specific for RNR2, SUC2, ANB1, MFA1, and RPS13 (which serves as an internal control). Expression of RNR2 is identical in the two internal deletions and the strain that contains only vector sequences (not shown). The TR1 and ANB1 transcripts are not related. contains the minimal Tupl-interaction domain (TPRl3), fully represses $M F A 1$ but does not affect expression of the other genes. Thus, specific repression functions of Cyc8 are progressively abolished as sequences are deleted from the carboxyl terminus.

The simplest explanation for these observations is that distinct subsets of TPR motifs are required for repression of the different classes of genes affected by the Cyc8Tup1 corepressor. Alternatively, the different classes of genes might simply require quantitatively different amounts of a common TPR function. To distinguish between these possibilities, we analyzed two internal deletions, $\Delta 175-281$ and $\Delta 175-304$, which lack TPR4-7 and TPR4-8, respectively (Figs. 1 and 3). Both of these derivatives contain the Tup 1 -interaction domain, and as expected, they repress the transcription of $M F A 1$. Neither derivative can repress $A N B 1$, but interestingly, the $\Delta 175-281$ (but not the $\Delta 175-304$ ) derivative represses transcription of SUC2 completely. Thus, with respect to repression of ANB1 and SUC2, the $\triangle 175-281$ and N301 derivatives have opposite phenotypes.

These results suggest that besides Tupl and the Tup1interaction domain of $\mathrm{Cyc} 8$, discrete combinations of TPR motifs of Cyc 8 are employed for repression of spe- cific sets of promoters. TPR8, TPR9, and possibly TPR10, but not the region containing TPR4-7, are required for glucose repression. In contrast, TPR4-7, but not TPR8-10, are required for oxygen repression. Finally, repression of a-specific genes requires only the Tuplinteraction domain of Cyc8 (TPR1-3), whereas repression of DNA damage-regulated-genes appears to require the entire TPR domain and more carboxy-terminal sequences.

\section{Functional interaction between Mig1} and the Cyc8-Tup1 corepressor

Mig1, a zinc finger repressor protein that binds to the promoters of SUC2 and other glucose-repressible genes (Nehlin and Ronne 1990), has been proposed to be a target of the Cyc8-Tupl corepressor complex (Keleher et al. 1992). To test this hypothesis, we examined whether the transcriptional repression by a LexA-Migl hybrid protein was affected by Cyc8 and Tup1 (Table 3). As shown previously for LexA-Cyc8 and LexA-Tup1 (Table 2; Tzamarias and Struhl 1994; Keleher et al. 1992), LexA-Mig1 represses transcription in a Cyc8- and Tup1-dependent manner from a promoter containing LexA operators up-

Table 3. Transcriptional repression by LexA-Mig1

\begin{tabular}{|c|c|c|c|c|c|c|c|c|c|}
\hline \multirow{3}{*}{$\begin{array}{l}\text { LexA hybrid } \\
\text { proteins }\end{array}$} & \multirow{2}{*}{\multicolumn{3}{|c|}{ Fold repression }} & \multicolumn{6}{|c|}{ Promoter (strain) } \\
\hline & & & & \multirow{2}{*}{$\begin{array}{l}\text {-Lop } \\
\text { (Glu) }\end{array}$} & \multirow{2}{*}{$\begin{array}{l}4 \text { Lop } \\
\text { (Glu) }\end{array}$} & \multirow{2}{*}{$\begin{array}{l}\text { - Lop } \\
(\text { cyc8) }\end{array}$} & \multirow{2}{*}{$\begin{array}{l}4 \text { Lop } \\
\text { (cyc8) }\end{array}$} & \multirow{2}{*}{$\begin{array}{l}\text {-Lop } \\
\text { (tup1) }\end{array}$} & \multirow{2}{*}{$\begin{array}{l}4 \text { Lop } \\
\text { (tup1) }\end{array}$} \\
\hline & (Glu) & $($ cyc8) & (tup1) & & & & & & \\
\hline LexA-Mig1 & 3.9 & 0.6 & 0.7 & 93 & 24 & 39 & 61 & 52 & 74 \\
\hline LexA & 1.2 & 1.1 & 1.3 & 100 & 80 & 55 & 50 & 90 & 70 \\
\hline Vector & 1.2 & 1.0 & 1.3 & 100 & 85 & 55 & 55 & 90 & 70 \\
\hline
\end{tabular}

$\beta$-Galactosidase activities (average of three independent transformants) of wild-type, cyc8, or tup1 strains grown in glucose medium that harbor the indicated promoters and proteins. Values are normalized to $A_{600}$ of cells at the time of collecting and are accurate to $\pm 30 \%$. Fold repression represents the ratio of $\beta$-galactosidase activities in strains containing plasmids that either lack (-Lop) or contain four LexA operators (4 Lop) upstream of the CYC1 promoter fused to the lacZ structural gene. 
stream of the cyc1 UAS and TATA elements. However, the repressive effect of LexA-Migl (4-fold) is considerably less than that of LexA-Cyc8 (27-fold) or LexA-Tup1 (15-fold) when compared on the same promoter. Unexpectedly, when tested on a minimal promoter consisting of four LexA operators upstream of a GAL1 TATA element, LexA-Migl (but not LexA) activates transcription in $c y c 8$ and tup1 deletion strains but not in an isogenic wild-type strain (Table 4). These results define a functional interaction between Mig1 and the Cyc8-Tup1 corepressor that is likely to be involved in repression of glucose-regulated genes. Furthermore, they suggest that Cyc8-Tup1 can function both as a corepressor and as an inhibitor of Migl activation. Similar conclusions concerning the relationship between Mig1 and Cyc8-Tupl have been obtained by Treitel and Carlson.

The Cyc8-interaction and WD domains of Tup1 are differentially required for repression of specific promoters

We have shown previously that Tup1-N200, a derivative containing the Cyc8-interaction and transcriptional repression domains but lacking the region containing the six WD motifs, carries out many functions of the wildtype Tupl protein (Tzamarias and Struhl 1994). In particular, Tup1-N200 partially represses SUC2 and ANB1 transcription, and it rescues the slow growth and clumpy phenotype of a tup1 deletion strain. Tup1 derivatives lacking either the repression domain (e.g., N72) or the Cyc8-interaction domain (e.g., C565, C425) are unable to repress SUC2 and ANB1 transcription.

We extended this analysis by assaying the same Tupl derivatives for expression of MFA1 and RNR2 (Fig. 4). As expected, Tup1 derivatives containing only the Cyc8interaction domain (e.g., N72) or the region containing the six WD motifs (C324) fail to repress any of the four genes. However, in contrast to its effects on SUC2, $A N B 1$, and cell growth and morphology, Tup1-N200 fails to repress $M F A 1$ and $R N R 2$ transcription, indicating that additional regions of Tupl are required for repression of genes regulated by cell type and DNA damage. In addition, Tup1 derivatives lacking the Cyc8-interaction domain (Tup1-C565 and Tup1-C425) partially repress

Table 4. Transcriptional activation by LexA-Mig1 in cyc8 and tupl strains

\begin{tabular}{lccr}
\hline & \multicolumn{3}{c}{ Strains } \\
\cline { 2 - 4 } LexA proteins & WT & cyc8 & tup1 \\
\hline LexA-Mig1 & 3 & 21 & 17 \\
LexA & 4 & 5 & 5 \\
\hline
\end{tabular}

$\beta$-Galactosidase activities (average of three independent transformants) of wild-type (WT), cyc8, or typ1 deletion strains expressing the indicated proteins. The lacZ reporter plasmid contains four LexA operators upstream of the GAL1 TATA element. Values are normalized to $A_{600}$ of cells at the time of collecting and are accurate to $\pm 30 \%$.

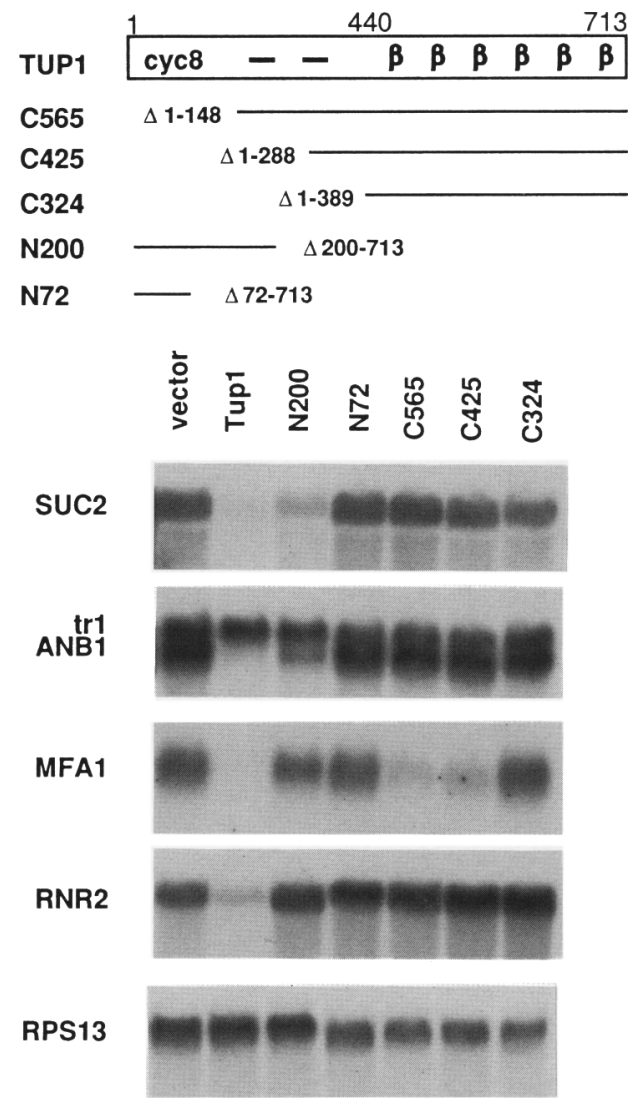

Figure 4. Repression of SUC2, ANB1, RNR2, and MFA1 by various Tupl derivatives. The three functional domains of Tupl, Cyc8-interaction domain (cyc8), independent repression domains $(-1$, and WD motifs $(\beta)$ are indicated along with the structure of the Tupl-deleted derivatives (named according to the amino- or carboxy-terminal sequence that it contains). RNA from tup 1 yeast strains expressing the indicating Tupl derivatives was fractionated in $1.4 \%$ agarose-formaldehyde gel, transferred to nylon membrane, and hybridized with ${ }^{32} \mathrm{P}$-labeled probes specific for $S U C 2, A N B 1, M F A 1, R N R 2$, and the internal RPS13 control.

the cell type-specific gene MFA1 but do not affect transcription of SUC2, ANB1, or RNR2. The distinct roles of Cyc8 and Tup 1 in repression of MFA1 and SUC2 are also observed in a comparison between cyc 8 and tup 1 deletion strains (Fig. 3). SUC2 levels are notably higher in a $c y c 8$ strain as compared with a tup1 strain, whereas the reverse is true for MFA1; levels of $A N B 1$ and RNR2 are comparable in both strains. Our results on MFA1 are in accord with recent observations that interaction of the Tup1 WD motifs with $\alpha 2$ is necessary for repression of a-specific genes (Komachi et al. 1994).

\section{Tup1 forms multimers}

In the course of testing LexA-Cyc8 derivatives for their ability to interact with Tup1-N72-VP16 in the two-hybrid assay, we examined the possibility that Tup1 might self-associate (Table 5). In combination with Tup1-N72- 
Table 5. Two-hybrid assays for Tup1-Tup1 interaction

\begin{tabular}{|c|c|c|c|c|c|c|c|c|c|c|c|c|}
\hline \multirow{3}{*}{$\begin{array}{l}\text { LexA hybrid } \\
\text { proteins }\end{array}$} & \multirow{2}{*}{\multicolumn{3}{|c|}{ Fold activation }} & \multicolumn{9}{|c|}{ VP16 hybrid proteins/strain background } \\
\hline & & & & \multirow{2}{*}{$\begin{array}{c}\mathrm{V} \\
\text { (WT) }\end{array}$} & \multirow{2}{*}{$\begin{array}{c}\mathrm{T} \\
(\mathrm{WT})\end{array}$} & \multirow{2}{*}{$\begin{array}{c}\text { TV } \\
\text { (WT) }\end{array}$} & \multirow{2}{*}{$\begin{array}{c}\mathrm{V} \\
(\mathrm{cyc} 8)\end{array}$} & \multirow{2}{*}{$\begin{array}{c}\mathrm{T} \\
\langle\text { cyc8 }|\end{array}$} & \multirow{2}{*}{$\begin{array}{c}\mathrm{TV} \\
|c y c 8|\end{array}$} & \multirow{2}{*}{$\frac{\mathrm{V}}{(\operatorname{tup} 1)}$} & \multirow{2}{*}{$\underset{\mid \operatorname{tup} 1)}{\mathrm{T}}$} & \multirow{2}{*}{$\begin{array}{c}\mathrm{TV} \\
\text { |tup1 }\end{array}$} \\
\hline & (WT) & (cyc8) & $\langle$ tup1) & & & & & & & & & \\
\hline Tup1 & 16 & 8.5 & 6.0 & 3 & 3 & 47 & 2 & 2 & 17 & 4 & 2 & 24 \\
\hline Tup1-C565 & 0.7 & 1.3 & 0.7 & 3 & 4 & 3 & 3 & 3 & 4 & 2 & 3 & 2 \\
\hline Tup1-N200 & 60 & 22 & 45 & 5 & 7 & 420 & 3 & 2 & 44 & 3 & 4 & 180 \\
\hline Tupl-N72 & 20 & 5.5 & 16 & 4 & 7 & 140 & 4 & 4 & 22 & 3 & 5 & 81 \\
\hline LexA & 1.0 & 1.5 & 1.0 & 2 & 5 & 5 & 2 & 2 & 3 & 3 & 3 & 3 \\
\hline
\end{tabular}

$\beta$-Galactosidase activities (average of three independent transformants) of wild-type (WT), cyc8, or tup1 strains expressing the indicated proteins [(V) VP16; (T) Tupl; (TV) Tup1-VP16]. The lacZ reporter plasmid contains four LexA operators upstream of the GAL1 TATA element. Values are normalized to $A_{600}$ of cells at the time of collecting and are accurate to $\pm 30 \%$. Fold activation represents the ratio of $\beta$-galactosidase activities in strains containing Tupl-VP16 vs. those containing Tup1.

VP16, LexA-Tupl stimulates transcription 16-fold, and smaller amino-terminal derivatives (LexA-Tup1-N200 and LexA-Tupl-N72) are equally or more efficient. These two-hybrid interactions occur in cyc8 and tup1 deletion strains, although to a slightly lesser extent in some cases, indicating that they do not require the chromosomal copies of TUP1 and CYC8. In contrast, carboxy-terminal LexA-Tupl derivatives that lack 148 or more amino-terminal amino acids do not stimulate transcription above the background level. Thus, the 72 amino-terminal residues of Tupl are necessary and sufficient for homomultimerization in vivo. Interestingly, the same region of Tup 1 interacts directly with Cyc8 (Tzamarias and Struhl 1994).

To test whether Tup1 multimerization occurs in the absence of additional yeast proteins, we incubated ${ }^{35} \mathrm{~S}$ labeled Tup1-N253 protein with agarose beads containing GST-Tup1-N250, GST-Tup1-C324, and GST alone. As seen in Figure 5, Tup1-N253 strongly associates with GST-Tupl-N250 but not with GST alone or GST-

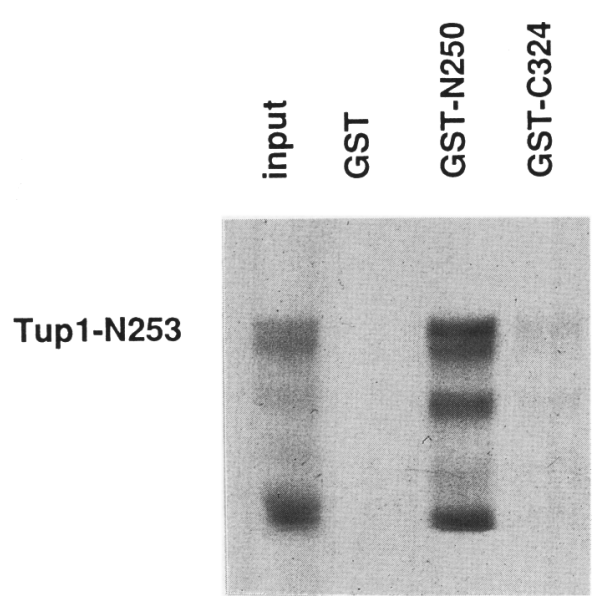

Figure 5. Tupl-Tupl interaction in vitro. ${ }^{35}$ S-Labeled TuplN253 stably bound to agarose beads containing GST, GSTTup1-N250, and GST-Tup1-C324. The lane labeled input contains only $20 \%$ of the amount of the protein that was incubated with the beads.
Tup1-C324. These results indicate that Tup1 forms multimers through the $\mathrm{Cyc} 8$-association domain (amino acids $1-72$ ) but in a manner independently from $\mathrm{Cyc} 8$, suggesting the possibility that Cyc8 associates with a dimeric (or higher order) form of Tupl.

\section{Discussion}

\section{A specific set of Cyc8 TPR motifs interact directly with Tup1}

TPR motifs have been found in a wide variety of eukaryotic proteins, and they have been proposed to form amphipathic $\alpha$-helices that mediate protein-protein interactions (Hirano et al. 1990; Goebl and Yanagida 1991). The biochemical functions of TPR motifs are generally unknown. In the best-studied case, the TPR-containing proteins Cdc16, Cdc23, and Cdc27 form a complex essential for mitosis (Lamb et al. 1994). Mutations in the most conserved TPR motif of $\mathrm{Cdc} 27$ reduce the interaction (direct or indirect) with $\mathrm{Cdc} 23$ but not with $\mathrm{Cdc} 16$ or Cdc27 itself. Although this observation could be interpreted in terms of a specific role for this TPR motif in the formation of complexes containing $\mathrm{Cdc} 27$ and Cdc23, such complexes might simply require more TPR motifs than complexes containing $\mathrm{Cdc} 27$ and $\mathrm{Cdc} 16$ or Cdc27 multimers. Furthermore, it is unknown whether TPRs in Cdc27, Cdc23, and Cdc16 directly interact with each other or with other proteins.

In this paper we demonstrate that TPR motifs can directly mediate protein-protein interactions. Specifically, a region of the Cyc8 TPR domain interacts with Tup1 in vitro in the absence of yeast proteins (Fig. 2). In contrast to previous expectations (Hirano et al. 1990; Sikorski et al. 1990; Goebl and Yanagida 1991), the region of Tup1 that interacts with the Cyc8 TPR motifs does not contain TPR or WD motifs. However, the sequence of this Tup1 region (residues 1-72) is compatible with $\alpha$-helix formation, suggesting the possibility that the Cyc8Tup1 interaction is mediated by interacting $\alpha$-helices. Given that Tupl residues 1-72 also self-associate in vitro (Fig. 5), the Cyc8-Tup1 complex might involve a three (or more)-stranded $\alpha$-helical coiled coil (Harbury et al. 1994). 
The Cyc8-Tupl interaction is mediated by a specific combination of TPR motifs. TPR 1-3 interact efficiently with Tup1, whereas derivatives with more TPR motifs (e.g. TPR2-7) do not. Thus, despite the primary sequence similarity between individual repeats, the TPR motifs of Cyc8 are not functionally redundant. Consistent with this idea, TPR4-7 and TPR8-10 are functionally distinct with respect to glucose and oxygen repression. It is likely that TPR4-7 and TPR8-10 interact with distinct, although as yet unidentified, proteins. Taken together, our results suggest that TPR motifs represent a basic structural scaffold that accommodates a variety of protein surfaces that specifically interact with other proteins. In this view the highly conserved residues in TPR motifs are likely to be involved in the basic structure, with less conserved residues being important in determining specificity.

\section{Differential recruitment of the Cyc8-Tup1 complex to pathway-specific promoters}

The Tupl repression domain mediates the transcriptional inhibitory function of the Cyc8-Tupl corepressor and, hence, is required for all known functions of the complex (Fig. 4; Tzamarias and Struhl 1994). As a consequence, the Tupl-interaction domain of Cyc8 (TPR 13 ) is essential for all known Cyc8 functions. Cyc8 derivatives that lack this domain fail to repress genes regulated by cell type, glucose, oxygen, and DNA damage (Fig. 3), and they do not rescue any of the physiological defects of a cyc8 deletion strain such as slow growth, temperature-sensitive lethality, and aberrant colony morphology. Furthermore, these Cyc8 derivatives fail to repress the transcription when they are bound to DNA via the LexA DNA-binding domain.

Although the Tupl repression domain inhibits transcription when artifically tethered upstream of a promoter, repression of natural promoters requires recruitment of the Cyc8-Tup1 complex through other proteinprotein interactions. We provide strong evidence that Cyc8 plays an important role in differential recruitment of Cyc8-Tup1 to pathway-specific promoters. Two specific combinations of TPRs, along with but distinct from the Tupl-interaction region, are required for repression of SUC2 and ANB1. TPR8-10 are specifically required for glucose repression of $S U C 2$, whereas TPR4-7 are required specifically for oxygen repression of $A N B 1$. However, these TPR motifs are dispensable, and the Tuplinteraction domain (TPR $1-3$ ) is sufficient, for Tup1-dependent repression by LexA-Cyc8.

From these observations, we propose that TPR4-7 and TPR8-10 mediate distinct protein-protein interactions that recruit the co-repressor complex to oxygen- and glucose-repressible promoters, respectively (Fig. 6). In the simplest model for this recruitment, the relevant TPR motifs of Cyc8 would directly interact with pathwayspecific, DNA-binding proteins such as Migl and probably Rox1. Alternatively, the Cyc8 TPR motifs might interact with intermediary proteins that interact with Migl or Roxl. Affinity chromatography experiments of
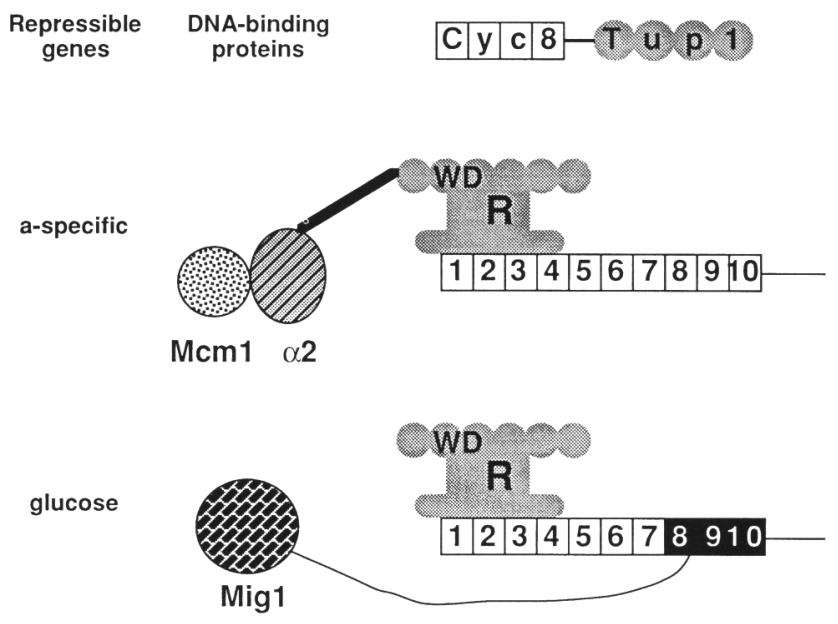

oxygen

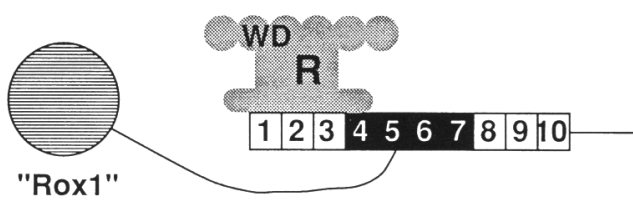

DNA-damage

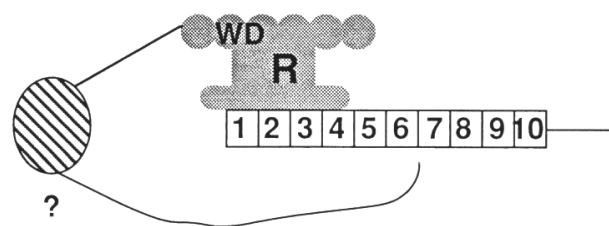

Figure 6. Model for differential recuitment of Cyc8-Tupl to pathway-specific promoters. For each class of repressible gene, interactions between the pathway-specific, DNA-binding proteins and the Cyc8-Tupl complex are indicated. Repression of a-specific genes involves a direct interaction (thick line) between $\alpha 2$ and the WD domain (six copies indicated by small, shaded circles) of Tup1 (Komachi, 1994). Repression of glucoseregulated genes involves a functional interaction (thin line) between Migl and Cyc8 TPR8-10 (blackened). Repression of oxygen-regulated genes involves a functional interaction (thin line) between Rox1 and Cyc8 TPR4-7 (blackened); the evidence that Roxl is the DNA-binding protein that responds to Cyc8Tupl is suggestive but not conclusive (indicated by quotation marks around Rox1). Repression of DNA damage-regulated genes involves functional interactions (thin lines) between an unknown DNA-binding protein and both the WD domain of Tupl and unspecified regions of Cyc8. Each of the above functional interactions might reflect direct protein-protein contact and/or interactions with other proteins. Other interactions besides those indicated may also be important for recruitment. Formation of the Cyc8-Tupl complex requires an amino terminal region of Tupl (thin gray oval) and TPR1-3 of Cyc8; it is likely that the complex contains multiple Tupl molecules. In all cases, transcriptional repression is mediated by a specific region of Tup1 (shaded box labeled R). (See text for details.)

the type used to characterize the Cyc8-Tup1 interaction have revealed only a tenuous Cyc8-Roxl interaction and no detectable Cyc8-Migl interaction even in the pres- 
ence of Tupl (D. Tzamarias and K. Struhl, unpubl.). Finally, whereas our results clearly implicate TPR4-6 and TPR8-10 in recruitment of the Cyc8-Tupl complex to these two sets of promoters, they do not exclude possible contributions of Tupl and/or the Tup1-interaction domain of Cyc8.

In contrast to repression of glucose- and oxygen-regulated genes, recent results indicate that recruitment of the Cyc8-Tupl corepressor to cell type specific genes is mediated by a direct interaction between $\alpha 2$ and the WD motifs of Tup1 (Komachi et al. 1994). Moreover, several results indicate that cell type-regulated genes can be partially repressed in the absence of Cyc8. First, the derepression level of a-specific genes such as MFA1 (Fig. 3) and STE6 (Cooper et al. 1994) is significantly lower in a cyc8 deletion strain than in an isogenic tup1 deletion strain. Second, Tupl derivatives lacking the Cyc8-interaction domain but containing the WD and repression domain partially repress MFA1 transcription (Fig. 4). Third, the only region of $\mathrm{Cyc} 8$ necessary for full repression of MFA1 is the Tup1-interaction domain (TPR1-3), which is not sufficient for repression of any other pathway-specific gene tested (Fig. 3). Thus, Cyc8 plays only an auxiliary role in repression of cell type genes, unlike its essential role in repression of glucose- and oxygenregulated genes. In this auxiliary role, TPR $1-3$ of Cyc 8 might stabilize a multimeric form of Tupl or mediate additional interactions that strengthen the association of the Tup1 WD motifs with $\alpha 2$.

Repression of $R N R 2$, a gene induced by DNA damage, is distinct from repression of SUC2, ANB1, and MFA1 in that it requires the entire TPR domain of Cyc8. Moreover, unlike the case for SUC2 and ANB1, repression of $R N R 2$ is abolished in Tupl derivatives lacking the WD domain. Thus, recruitment of the corepressor complex to DNA damage-inducible promoters may be mediated by the cooperative function of both the TPR domain of Cyc8 and the WD domain of Tupl.

Taken together, our results and those of Komachi et al. (1994) indicate that the Cyc8-Tup1 corepressor complex is differentially recruited to pathway-specific promoters (Fig. 6). We suggest that the underlying mechanism of this differential recruitment is that specific combinations of Cyc8 TPR motifs and the Tupl WD domain possess distinct protein-protein interaction specificities. In this view different surfaces of the Cyc8-Tupl complex interact (directly or indirectly) with the pathwayspecific repressors, whose DNA-binding domains are structurally dissimilar $(\alpha 2$ contains a homeo domain, Migl contains a zinc finger, and Roxl contains an HMG motif). Although the Cyc8-Tup1 complex appears to be differentially recruited to promoters regulated by glucose, oxygen, cell type, and DNA damage, we presume that transcriptional inhibition is mediated by the Tup 1 repression domain in all cases.

\section{Cyc8-Tup1 can also inhibit the function of specific activation domains}

As proposed initially (Keleher et al. 1992), the pathway- specific, DNA-binding proteins play a passive role in the repression process, serving merely to recruit Cyc8-Tupl to the relevant promoters. This view was based on the following observations on repression of cell type-specific genes. First, comparable expression levels are observed in strains lacking functional operators $(\alpha 2-\mathrm{Mcml}$ or al$\alpha 2)$, DNA-binding proteins $(\alpha 2$, a1), or the Cyc8-Tup1 complex. Second, when tested upstream of a heterologous TATA element, the $\alpha 2-\mathrm{Mcm} 1$ operator behaves as a very weak activator (because of $\mathrm{Mcm}$ 1, which can bind the operator in the absence of $\alpha 2$ ) that is unaffected by loss of Cyc8 function (Keleher et al. 1992). Whereas $\alpha 2$ plays a passive role in repression, it is unknown whether this is the case for other DNA-binding proteins that are targets of the Cyc8-Tupl corepressor.

The fact that transcriptional activity of LexA-Mig1 depends on Cyc8-Tup1 function demonstrates that Migl is a target of the Cyc8-Tupl corepressor complex. Furthermore, LexA-Mig1 represses transcription when bound upstream of an intact promoter (Table 3; Treitel and Carlson 1995), suggesting that recruitment of Cyc8Tupl plays a role in Mig1-dependent repression. However, LexA-Mig1 behaves as a transcriptional activator in cyc8 and tup1 strains (Table 4; Treitel and Carlson 1995), suggesting that Cyc8-Tup1 can also function as an inhibitor of Migl transcriptional activity, possibly by interacting with and, hence, masking an activation domain in Mig1. Thus, in addition to acting as a general repressor of transcription that is recruited to particular classes of promoters, Cyc8-Tupl can inhibit the function of specific activator proteins.

\section{Materials and methods}

Expression of Cyc8 and Tup1 derivatives in yeast

The TRP1 centromeric vector YCp91, which was used for expression of all Cyc8, Tupl, and LexA derivatives, contains the $A D H 1$ promoter and 5 '-untranslated sequence (including the ATG start codon), following by sequences encoding the SV40 nuclear localization signal and the HAl epitope from the influenza virus (NLS-Flu), a polylinker, three stop codons (in all three frames), and a 410-bp fragment containing the CYC8 termination region (Tzamarias and Struhl 1994). The entire CYC8coding sequences from an artificial BamHI site inserted 9 bp upstream of the ATG initiation codon to the AseI located $20 \mathrm{bp}$ downstream of the stop codon were cloned between BamHI and Asp 718 sites. Cyc8 deletion derivatives containing 100, $175,301,353,597$, and 816 amino-terminal residues were constructed in the same manner by inserting BamHI-PstI, BamHI-BstXI, BamHI-Asp718, BamHI-MluI, BamHI-PvuII, and $B a m H I-A l w N I$, respectively. To generate molecules containing Cyc8 residues $79-300$ and $113-300$, SmaI sites were introduced at positions 79 and 113 by PCR, and the resulting SmaI-Asp718 fragments were cloned into the YCp91 expression vectors. Internally, deletions $(\Delta 175-281$ and $\Delta 175-304)$ were constructed by using $\mathrm{Bal} 31$ to remove the amino-terminal 281 and 304 residues and then ligating the resulting molecules in-frame with a DNA fragment encoding amino acid sequence 1-174 (BamHI-BstXI fragment).

YCp91 molecules expressing Tupl and LexA-Tupl derivatives have been described previously (Tzamarias and Struhl 1994). The hybrid Tup1N72-VP16, which was used in the two- 
hybrid experiments, contains a SmaI-MluI fragment of Tup1 (amino acids 1-72) fused in-frame to a $B g I I I-B a m H I$ fragment containing the activation domain of VP16 (amino acids 414 553); control plasmids contain either the Tup1-N72 or the VP16 fragment. All three fragments were inserted in the YEp92, which contains the expression casette of YCp91 in the LEU2 multicopy plasmid YEpLac181 (Tzamarias and Struhl 1994). To generate a plasmid expressing LexA-Migl, a BamHI-Asp718 DNA fragment containing the entire Migl-coding region from -6 to +1514 was cloned in YCp91.

\section{Transcriptional repression and two-hybrid assays in vivo}

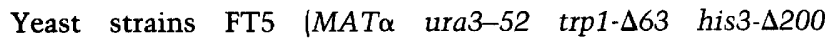
leu2::PET56) and derivatives containing the tup1 $1:$ HIS3 (remove Tupl codons -2 to 672 ) and cyc8- $\triangle 9:: H I S 3$ (removes Cyc8 codons 99-862) alleles have been described previously (Tzamarias and Struhl 1994). For two-hybrid assays, strains contained the URA3 multicopy plasmid JK103, in which the $l a c Z$ gene is driven by a promoter with four LexA operators upstream from the GAL1 TATA element (Kamens et al. 1990). The lacZ reporter constructs used in the LexA-dependent repression assay were derived from the plasmids pLG $\Delta 312 S$ and JK1621 and integrated at the URA3 locus (Tzamarias and Struhl 1994). pLG $\triangle 312 S$ contains a $C Y C 1$ fragment $(-324$ to +141$)$, including the two UASs and TATA region (Guarente and Mason 1983). JK1621 is a derivative pLG $\Delta 312 \mathrm{~S}$ with an insertion of four LexAbinding sites at a SmaI site upstream of the two UASs (Keleher et al. 1992).

$\beta$-Galactosidase assays were performed on yeast cells that were harvested in early $\log$ phase $\left(A_{600}<1.0\right)$ and then washed in $20 \mathrm{~mm}$ Tris (pH 7.5), $10 \mathrm{mM}$ EDTA to disperse the clumpy cyc8 and tup 1 cells. Cells were grown in glucose medium containing casamino acids and uracil. The numbers in the tables are normalized to $A_{600}$ and represent the average of at least three independent transformants; they are accurate to $\pm 30 \%$.

Repression of natural yeast genes was analyzed by RNA blotting. Total RNAs from appropriate strains grown in glucose medium containing casamino acids and uracil were fractionated in a $1.4 \%$ agarose gel containing $5.5 \%$ formaldehyde, transferred to nitrocellulose, and hybridized to ${ }^{32} \mathrm{P}$-labeled probes that were generated by nick translation or 5'phosphorylation of the following DNAs: $1.2 \mathrm{~kb}$ HindIII fragment from $S U C 2$; 1.5kb SmaI-BamHI fragment containing the entire ANB1 gene (which also hybridizes with a second transcript that is indicated tr1); 1-kb internal HindIII fragment from RNR2, oligonucleotide complementary to the MFA1-coding strand (codons 21-35); and 450-bp HindIII-EcoRI fragment from RPS13, which encodes a ribosomal protein and serves as an internal control.

\section{In vitro interaction assay}

GST hybrid proteins from $500 \mathrm{ml}$ of exponentially growing Escherichia coli (strain DH5a) cells with the appropriate plasmids that were induced with $0.25 \mathrm{~mm}$ IPTG for $3 \mathrm{hr}$ at $30^{\circ} \mathrm{C}$, harvested by centrifugation, and frozen immediately. The bacterial pellets were resuspended in $5 \mathrm{ml}$ of a buffer containing $100 \mathrm{mM} \mathrm{NaCl}, 20 \mathrm{~mm}$ HEPES ( $\mathrm{pH} 7.5$ ), 1 mM DTT, 1 mM EDTA, $1 \%$ Triton, $20 \%$ glycerol, $0.5 \%$ BSA, and protease inhibitors $\mid 1$ $\mathrm{mM}$ PMSF, $5 \mathrm{mg} / \mathrm{ml}$ of leupeptin, and $5 \mathrm{mg} / \mathrm{ml}$ of pepstatin) and sonicated (six strokes, $10 \mathrm{sec}$ each) at $4^{\circ} \mathrm{C}$. Cell debris was removed by centrifugation at $10,000 \mathrm{~g}$ for $15 \mathrm{~min}$, and the supernatant was incubated with an equal volume $(0.5-2.0 \mathrm{ml}$, depending on protein yield) of glutathionine-agarose beads (activated according to instructions given by Sigma) for $1 \mathrm{hr}$ at $4^{\circ} \mathrm{C}$ with rocking. The protein-containing beads were washed four times with 10 volumes of the same buffer (without BSA) and stored at $4^{\circ} \mathrm{C}$.

${ }^{35}$ S-labeled proteins were synthesized in vitro, using $\mathrm{T} 3$ or $\mathrm{T} 7$ RNA polymerase and rabbit reticulocyte lysate in a $40 \mu \mathrm{l}$ reaction according to the manufacturer (Promega). Five to $10 \mu \mathrm{l}$ of ${ }^{35} \mathrm{~S}$-labeled protein was incubated with $1-2 \mu \mathrm{g}$ of agarose beadbound GST hybrid protein in $400 \mu \mathrm{l}$ reaction containing $100 \mathrm{~mm}$ $\mathrm{NaCl}, 20 \mathrm{~mm}$ Tris- $\mathrm{HCl}(\mathrm{pH} 8.0), 0.1 \% \mathrm{NP}-40$, and $0.25 \% \mathrm{BSA}$ (plus protease inhibitors) at $4^{\circ} \mathrm{C}$ for $2 \mathrm{hr}$ with rocking. The agarose beads were washed three times with $1.5 \mathrm{ml}$ of interaction buffer and one more time with the same buffer lacking BSA. The bound proteins were eluted in buffer containing SDS and then fractionated by SDS-PAGE.

\section{Acknowledgments}

We thank Marian Carlson for communicating results prior to publication and Steve Elledge for RNR2 plasmids. This work was supported by a postdoctoral fellowship from the Human Frontiers of Science Program (D.T.) and by research grant GM30186 to K.S. from the National Institutes of Health.

The publication costs of this article were defrayed in part by payment of page charges. This article must therefore be hereby marked "advertisement" in accordance with 18 USC section 1734 solely to indicate this fact.

\section{References}

Auble, D.T., K.E. Hansen, C.G.F. Mueller, W.S. Lane, J. Thorner, and S. Hahn. 1994. Mot 1, a global repressor of RNA polymerase II transcription, inhibits TBP binding to DNA by an ATP-dependent mechanism. Genes \& Dev. 8: 1920-1934.

Balasubramanian, B., C.V. Lowry, and R.S. Zitomer. 1993. The ROX1 repressor of Saccharomyces cerevisiae hypoxic genes is a specific DNA-binding protein with a high-mobilitygroup motif. Mol. Cell. Biol. 13: 6071-6078.

Collart, M. and K. Struhl. 1994. NOT1(CDC39), NOT2(CDC36), NOT3, and NOT4 encode a global negative regulator of transcription that differentially affects TATA-element utilization. Genes \& Dev. 8: 525-537.

Cooper, J.P., S.Y. Roth, and R.T. Simpson. 1994. The global transcriptional regulators, SSN6 and TUP1, play distinct roles in the establishment of a repressive chromatin structure. Genes \& Dev. 8: 1400-1410.

Elledge, S.J., Z. Zhou, J.B. Allen, and T.A. Navas. 1993. DNA damage and cell cycle regulation of ribonucleotide reductase. BioEssays 15: 333-339.

Goebl, M. and M. Yanagida. 1991. The TPR snap helix: A novel protein repeat from mitosis to transcription. Trends Biochem. Sci. 16: 173-177.

Goutte, C. and A.D. Johnson. 1988. al protein alters the DNA binding specificity of $\alpha 2$ repressor. Cell 52: 875-882.

Grunstein, M. 1990. Histone function in transcription. Annu. Rev. Cell Biol. 6: 643-678.

Guarente, L. and T. Mason. 1983. Heme regulates transcription of the $c y c 1$ gene of $S$. cerevisiae via an upstream activation site. Cell 32: 1279-1286.

Harbury, P.B., P.S. Kim, and T. Alber. 1994. Crystal structure of an isoleucine-zipper trimer. Nature 371: 80-83.

Herschbach, B.M., M.B. Arnaud, and A.D. Johnson. 1994. Transcriptional repression directed by the yeast $\alpha 2$ protein in vitro. Nature 370: 309-311.

Hirano, T., N. Kinoshita, K. Morikawa, and M. Yanagida. 1990. Snap helix with knob and hole: essential repeats in S. pombe nuclear protein nuc2 $^{+}$. Cell 60: 319-328.

Inostroza, J.A., F.H. Mermelstein, I. Ha, W.S. Lane, and D. Re- 
inberg. 1992. Dr1, a TATA-binding protein-associated phosphoprotein and inhibitor of class II gene transcription. Cell 70: $477-489$.

Johnson, A.D. and I. Herskowitz. 1985. A repressor (MATa2 product) and its operator control expression of a set of cell type specific genes in yeast. Cell 42: 237-247.

Kamens, I., P. Richardson, G. Mosialos, R. Brent, and T. Gilmore. 1990. Oncogenic transformation by vRel requires an amino-terminal activation domain. Mol. Cell. Biol. 10: 2840-2847.

Keleher, C.A., C. Goutte, and A.D. Johnson. 1988. The yeast cell-type-specific repressor $\alpha 2$ acts cooperatively with a noncell-type-specific protein. Cell 53: 927-936.

Keleher, C.A., M.J. Redd, J. Schultz, M. Carlson, and A.D. Johnson. 1992. Ssn6-Tup1 is a general repressor of transcription in yeast. Cell 68: 709-719.

Komachi, K., M.J. Redd, and A.D. Johnson. 1994. The WD repeats of Tup 1 interact with the homeo domain protein $\alpha 2$. Genes \& Dev. 8: 2857-2867.

Lamb, J.R., W.A. Michaud, R.S. Sikorski, and P.A. Hieter. 1994. Cdc16p, Cdc23p and Cdc27p form a complex essential for mitosis. $E M B O$ J. 13: 4321-4328.

Mukai, Y., S. Harashima, and Y. Oshima. 1991. AAR1/TUP1 protein, with a structure similar to that of the $\beta$ subunit of $G$ proteins, is required for al $-\alpha 2$ and $\alpha 2$ repression in cell type control of Saccharomyces cerevisiae. Mol. Cell. Biol. 11: 3773-3779.

Nehlin, J.O. and H. Ronne. 1990. Yeast MIG1 repressor is related to the mammalian early growth response and Wilm's tumour finger proteins. EMBO I. 9: 2891-2898.

Paro, R. 1990. Imprinting a determined state into the chromatin of Drosophila. Trends Genet. 6: 416-421.

Schultz, J. and M. Carlson. 1987. Molecular analysis of SSN6, a gene functionally related to the SNF1 protein kinase of Saccharomyces cerevisiae. Mol. Cell. Biol. 7: 3637-3645.

Schultz, J., L. Marshall-Carlson, and M. Carlson. 1990. The $\mathrm{N}$-terminal TPR region is the functional domain of SSN6, a nuclear phosphoprotein of Saccharomyces cerevisiae. Mol. Cell. Biol. 10: 4744-4756.

Sikorski, R.S., M.S. Boguski, M. Goebl, and P. Hieter. 1990. A repeating amino acid motif in $C D C 23$ defines a family of proteins and a new relationship among genes required for mitosis and RNA synthesis. Cell 60: 307-317.

Struhl, K. 1985. Negative control at a distance mediates catabolite repression in yeast. Nature 317: 822-824.

Swanson, M.S. and F. Winston. 1992. SPT4, SPT5, and SPT6 interactions: Effects on transcription and viability in Saccharomyces cerevisiae. Genetics 132: 325-336.

Treitel, M.A. and M. Carlson. 1995. Repression by SSN6-TUP1 is directed by MIG1, a repressor/activator protein. Proc. Natl. Acad. Sci. 92: 3132-3136.

Trumbly, R.J. 1992. Glucose repression in the yeast Saccharomyces cerevisiae. Mol. Microbiol. 6: 15-21.

Tzamarias, D. and K. Struhl. 1994. Functional dissection of the yeast Cyc8-Tupl transcriptional corepressor complex. $\mathrm{Na}$ ture 369: 758-761.

Williams, F.E. and R.J. Trumbly. 1990. Characterization of TUP1, a mediator of glucose repression in Saccharomyces cerevisiae. Mol. Cell. Biol. 10: 6500-6511.

Williams, F.E., U. Varanasi, and R.J. Trumbly. 1991. The CYC8 and TUP1 proteins involved in glucose repression in Saccharomyces cerevisiae are associated in a protein complex. Mol. Cell. Biol. 11: 3307-3316.

Zitomer, R.S. and C.V. Lowry. 1992. Regulation of gene expression by oxygen in Saccharomyces cerevisiae. Microbiol. Rev. 56: 1-11. 


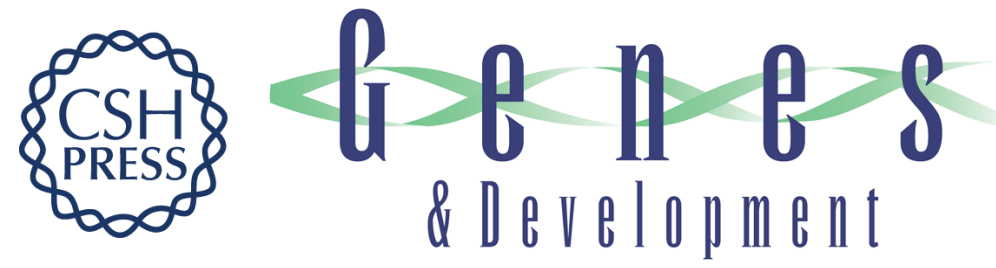

\section{Distinct TPR motifs of Cyc8 are involved in recruiting the Cyc8-Tup1 corepressor complex to differentially regulated promoters.}

D Tzamarias and K Struhl

\section{Genes Dev. 1995, 9:}

Access the most recent version at doi:10.1101/gad.9.7.821

References

License

Email Alerting

Service
This article cites 33 articles, 14 of which can be accessed free at:

http://genesdev.cshlp.org/content/9/7/821.full.html\#ref-list-1

Receive free email alerts when new articles cite this article - sign up in the box at the top right corner of the article or click here.

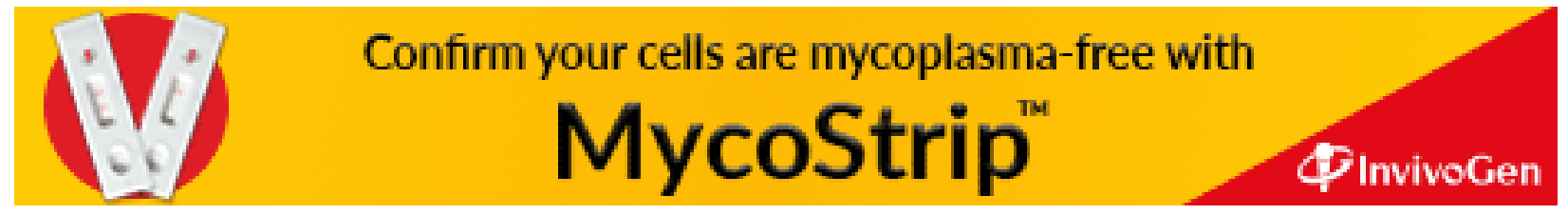

